\title{
'Pet scans': training dogs to sniff out disease
}

Clostridium difficile is a particularly troublesome 'superbug' that commonly affects people in healthcare facilities who have received antimicrobial medication. With the normal gut flora destroyed by antibiotic treatment, C. difficile quickly overruns the colon and releases toxins that cause bloating, diarrhea and abdominal pain. Left unchecked, C. difficile infection (CDI) can cause pseudomembranous colitis (a severe inflammation of the colon) and in rare cases will progress to a lifethreatening form of colonic swelling called toxic megacolon.

C. difficile is extremely transmissible and can quickly spread. Standard diagnostic tests for CDI are slow to produce results (cytotoxin assays requiring cell cultures) or are very expensive and require special equipment and expertise (nucleic amplification tests). The resultant delays in diagnosis allow ample time for $\mathrm{CDI}$ to become a ward-wide outbreak.

CDI-associated diarrhea has a characteristic scent. So distinct is the odor that nursing staff have been known to detect potential cases by smell. It was with this fact in mind that researchers at the VU University
Medical Center (Netherlands) enlisted the help of a beagle named Cliff.

In a proof of principle study, Marije Bomers and her colleagues examined whether a dog's superior sense of smell could be used to detect $C$. difficile in stool samples and hospital patients (BMJ published online 13 December 2012; doi:10.1136/bmj.e7396).

After two months of training, the dog was presented with two empirical tasks. First, Cliff was exposed to 100 stool samples (50 positive for $C$. difficile, 50 negative) and correctly identified all 50 positive samples and 47 of 50 negative samples $(100 \%$ sensitivity and $94 \%$ specificity). Cliff was then taken to two hospital wards to test his ability to detect $C$. difficile in patients. Here he correctly identified 25 of 30 cases (83\% sensitivity) and 265 of 270 controls ( $98 \%$ specificity).

The authors note that Cliff was quick and efficient, spending less than ten minutes with each patient. They also point out some limitations to using an animal as a diagnostic tool, including the potential to spread infection and the possible unpredictability in behavioral responses to other stimuli.

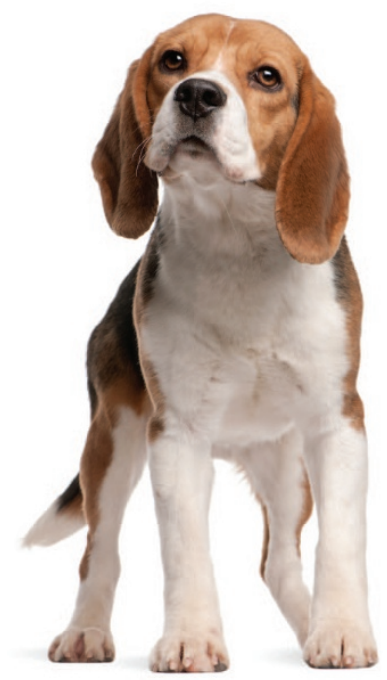

This study was the first to demonstrate that a dog can be used to detect C. difficile in stool samples and in patients. Cliff's diagnostic accuracy with the stool samples suggests that immediate CDI identification is achievable. To a slightly lesser degree, using dogs to identify CDI on the clinical ward may be possible too.

Thomas Rowley

\section{PREDICTING CARDIAC EVENT RISK IN SILICO}

Long QT syndrome type 1 (LQT1) is an inherited disorder in which mutations cause a loss of function of the gene KCNQ1.

Electrocardiography of affected individuals shows a longer than normal QT interval, representing a prolonged repolarization of the heart wall during the cardiac cycle. This prolongation results in a greater risk of developing arrhythmias and related cardiac events. Arrhythmia caused by mutations in KCNQ1 has been modeled in mice and in zebrafish and has been studied clinically, but attempts to identify the risk levels associated with specific mutations have so far been unsuccessful. Such a risk stratification would help to identify those who are most likely to experience cardiac events, guiding treatment strategies.

With this goal in mind, Coeli M. Lopes and Ilan Goldberg (University of Rochester School of Medicine and Dentistry, NY) worked with scientists at IBM campuses in NY and in Melbourne, Australia, to develop a computer model that predicts the effects of different KCNQ1 mutations on transmural repolarization potential (TRP) and their consequent risks for cardiac events. Model input included genetic and physiological data from 633 people with 34 different mutations leading to LQT1. The model itself comprised 192 cells, based on canine cardiac cells, that were assigned to one of three locations within the heart wall and were given different electrophysiological properties accordingly (J. Am. Coll. Cardiol. 60, 2182-2191; 2012).

The research team found that mutation-specific TRP was associated with an increased risk for cardiac events, including syncope, aborted cardiac arrest and sudden cardiac death. For every $10 \mathrm{~ms}$ of prolongation, risk of cardiac events increased by $35 \%$ and risk of life-threatening events increased by $27 \%$. The results suggest that the computer model can be used to predict clinical outcomes and improve risk assessment in people with LQT1. "Using this model, we can predict the likelihood that an individual will experience a deadly cardiac event based on the type of mutation they have and how that mutation acts," Lopes said in a press release.

Application of the model may be particularly valuable for people with mild to moderate QT prolongation, in whom QT interval measurements are less reliable for predicting cardiac events. In addition, the results identify four KCNQ1 mutations that are associated with the highest risk of cardiac events. The study is the first to use computer simulation to predict arrhythmia risk and may lead other researchers to apply more complex cardiac models to evaluate the effects of other genetic and lifestyle factors on heart rhythm. 